\title{
New Directions in Carbohydrate Engineering: A Metabolic Substrate-Based Approach to Modify the Cell Surface Display of Sialic Acids
}

BioTechniques 31:384-393 (August 2001)

\author{
Kevin J. Yarema \\ Johns Hopkins University \\ Baltimore, MD, USA
}

\section{INTRODUCTION}

Metabolic and cellular engineering are defined broadly as the "purposeful modification of intermediary metabolism" (5) and the "improvement of cellular activities" (2). Although these fields are highly multidisciplinary, they both share a heavy reliance on recombinant DNA technology. Recombinant DNA methods have proved particularly fruitful for industrial applications in bacteria and yeast; a growing number of these organisms are completely defined at the nucleotide level, and their genomes can be manipulated with relative ease. Unfortunately, this approach is less amenable to achieving precise modifications in the metabolic machinery or the macromolecular architecture of a human cell. In particular, the incorporation of xenobiotic compounds into cellular components by manipulation of the human genome or proteome is not trivial. This review describes an alternative, substrate-based approach in which novel cellular properties, in particular complex carbohydrates, are engineered by using analogs of smallmolecule metabolites instead of by the manipulation of the enzymes that process these compounds. tion with an externally delivered reagent. The sialic acid biosynthetic pathway is presented as a model system to illustrate both the practical aspects and theoretical considerations of a substrate-based cellular engineering approach. Specific applications of carbohydrate-based cell surface engineering include chemical construction of new glycosylation patterns on cells, new approaches to targeting tumor cell with either diagnostic or therapeutic agents, and installation of novel receptors on cells for facilitating viral-mediated gene delivery.

\section{INCORPORATION OF UNNATURAL METABOLIC INTERMEDIATES INTO THE CELLULAR ARCHITECTURE}

A substrate-based approach to cellular engineering consists of intercepting a biosynthetic pathway with an unnatural analog of a metabolic intermediate. The unnatural compound competes with the endogenous intermediate for biosynthetic incorporation into a cellular component by action of the enzymes that naturally occur in the cell (Figure 1). By selecting a substrate with the appropriate modification, the corresponding metabolic product can be engineered to contain a molecular handle capable of further elaboration with the appropriate chemical coupling partner. Consequently, the substratebased methodology presented in this paper can be considered a two-step approach (Figure 1): (i) biosynthetic incorporation of an unnatural metabolite into the architecture of a cell, (ii) followed by a subsequent chemical ligation reaction with an externally delivered reagent $(28,35,47,48)$.

An attractive feature of substratebased cellular engineering is the ability to mediate exact metabolic changes amidst an overwhelmingly complex cellular environment. By carefully selecting the properties of the unnatural metabolic intermediate, it is possible to target a particular biosynthetic pathway to the exclusion of all others. Such precision is a consequence of the stringent substrate specificity that most biosynthetic enzymes inherently possess to ensure the fidelity of biological processes. Indeed, this stringency often makes it more difficult to coax the target pathway to accept the altered metabolic intermediate than to avoid unwanted side reactions. Because the identification of a metabolic pathway permissive for unnatural substrates is not trivial, it is instructive to briefly consider a historical perspective to understand why recent advances in this field are focused largely on oligosaccharide engineering.

By the 1970s, it was firmly established that the surface of mammalian 
cells was richly decorated with a dense covering of complex oligosaccharides, collectively known as the glycocalyx. Even before many of the specific biological functions of these carbohydrates were elucidated, it was apparent that remodeling the molecular landscape of the cell surface transformed the behavior of cells $(1,15)$. Consequently, efforts were undertaken to modify the molecular nature of the plasma membrane by supplying cells with altered precursor monosaccharides in the hope that they would be metabolically incorporated into membrane components $(3,33,39)$. These early efforts generally did not result in the presentation of the unnatural precursors in cell surface glycoconjugates. Many of the compounds, however, did alter the molecular nature of the glycocalyx by inhibiting some or all of the natural glycosylation pathways of the cell (3). These changes, although largely nonspecific (and poorly characterized), nevertheless did have the desired result of modulating cell behavior, resulting in such changes as abnormal cellular growth patterns $(11,39)$. The tantalizing results of these early experiments intensified the search for monosaccharide precursors that would not only modify glycosylation patterns by inhibiting the expression of certain glycoforms but also would actually replace the normal sugars displayed on the cell surface $(10,33)$. Of particular interest, as discussed below, was the conversion of unnatural metabolic precursors into the corresponding cell surface-displayed sialic acids (Figure 2) (41).

Sialic acid is a generic term for a family of unique 9-carbon monosaccharides. The most abundant sialic acid in humans is $N$-acetyl neuraminic acid (NeuAc; Figure 2). When displayed on the cell surface, this sugar plays a defining role in several critical cellular processes including immunorecognition, cell adhesion and growth, and the binding of certain pathogens $(19,44)$. In addition, a number of human disease states, including cancer, are characterized by aberrant sialic acid expression (43). Considering that a primary factor when selecting a metabolic pathway for experimental intervention is to ensure that the proposed manipulation will im pact important cellular processes, the highly significant biological roles of sialic acid situates its biosynthetic pathway as an attractive model system to demonstrate the substrate-based approach to cellular engineering. An additional factor supporting the choice of this pathway as a model system is that it is fully defined at a molecular level (Figure 2). Finally, empirical evidence that the individual enzymes of the sialic acid pathway were permissive for altered substrates suggested that the entire pathway might also accommodate certain unnatural metabolic intermediates (10).

\section{DESIGN CONSIDERATIONS FOR ENGINEERING UNNATURAL METABOLIC INTERMEDIATES: THE SIALIC ACID PATHWAY AS A MODEL SYSTEM}

Once a biosynthetic pathway is chosen for experimental intervention, several features must be considered when designing an unnatural metabolic intermediate. The first decision is to select the point at which to intercept the pathway. In the example of the sialic acid pathway, each of the several intermediates (Figure 2) has the potential for replacement by an unnatural counterpart. Intercepting the pathway at a late stage has the advantage that fewer enzymes are required to process the unnatural compound into the final product. Considering that any modification of the natural substrate is likely to result in reduced processivity by each enzyme, interception of the pathway at a late stage is desirable. Unfortunately, the additional complexity of late intermediates requires more cumbersome synthetic routes to prepare analogs and also results in the increased lability of these compounds. In particular, the later intermediates in the sialic acid pathway such as CMP-NeuAc are unstable (Figure 2); they are also charged molecules unlikely to be membrane permeable and available to the target enzymes within a cell. These drawbacks necessitate intercepting the sialic acid pathway at a relatively early stage.

Careful analysis of the sialic acid pathway suggests that $\mathrm{N}$-acetylmannosamine (ManNAc; Figure 2) is the preferred step to introduce the unnatural metabolic intermediate. From a prac- tical viewpoint, ManNAc is a relatively simple compound, amenable to chemical manipulation. Furthermore, exogenous ManNAc can be uptaken by a cell, suggesting that an analog of this com pound should also be bioavailable (10, 30). As an additional benefit, ManNAc is the first committed intermediate in sialic acid biosynthesis. Therefore, unlike its immediate endogenous precursor UDP-GlcNAc, a compound utilized by several metabolic and biosynthetic pathways, ManNAc is destined almost exclusively for conversion to glycoconjugate-bound sialic acids. These factors imply that a ManNAc analog should be directed to a defined class of biological macromolecules, thereby effecting a precise change in a cellular property.

A second major consideration in substrate design is to ensure that the unnatural compound is chemically competent to undergo catalysis without simultaneously inhibiting metabolic flux through the pathway. A fluorinated ManNAc analog, $N$-trifluoroacetyl mannosamine (Figure 2), is a cautionary example of a metabolic intermediate that, although converted to its sialic acid counterpart, also reduced overall levels of cellular sialic acid and elicited significant toxicity at high micromolar concentrations (41). Considering that fluorinated compounds are often utilized as enzyme inhibitors because the chemical nature of the $\mathrm{C}-\mathrm{F}$ bond differs significantly from the natural $\mathrm{C}-\mathrm{H}$ bond, such deleterious effects on cellular metabolism are not unexpected. Clearly, care must be taken to design a compound that achieves a favorable balance between catalysis and inhibition. Specifically, elongating the $N$-acyl side chain of ManNAc with chemically inert alkyl substituents (Figure 2) suppressed inhibitory effects, allowing facile incorporation into cell surface sialic acids in human cells (21) and in vivo (18).

The third key design challenge is to prevent deleterious side reactions with other cellular components. Ideally, the metabolic analog must avoid both cross reactivity with the numerous other small-molecule metabolites within a cell and evade modification by non-target enzymes as well. Addressing the first point, care must be taken when modifying a natural substrate to ensure that it remains refractory to the wealth 
of potential reaction partners (hydroxyls, thiols, amides, esters, carboxylic acids, and so on) present in a cellular environment. As mentioned above, the first analogs successfully incorporated into the sialic acid pathway have the $N$-acyl side chain of ManNAc extended with inert alkyl substituents. This modification introduces no new chemical reactivity, thereby avoiding unwanted side reactions with the other chemical functionalities found within a cell. Second, a cell faced with the prospect of the introduction of a xeno- or abiotic compound has a number of defense mechanisms, including modification or conjugation by detoxification enzymes $(7,20)$, followed by release from the cell by the multidrug-resistant (MDR) protein family $(4,14)$. These issues mirror pharmacokinetic concerns that are reviewed adequately elsewhere $(6,31)$.

A final design consideration is to ensure that the incorporation of the unnatural moiety into the cellular architecture is of sufficient impact to modulate a cellular process. In the example of the sialic acid pathway, the biosynthetic incorporation of the $\mathrm{N}$-acyl-derivatized ManNAc derivatives into their cell surface sialic acid counterparts resulted in the extremely subtle modification of overall cell surface characteristics. Therefore, a fair question is whether this change can meaningfully alter a biological process. If it cannot, although the ability of the unnatural intermediate to navigate an entire metabolic pathway may be of utmost interest to the enzymologist, then it would be of minor relevance to the cellular engineer. As outlined below, the metabolic conversion of unnatural ManNAc analogs into their cell surface sialic acid counterparts has profound effects on biological processes, thereby validating the experimental utility of this approach.

\section{ENGINEERED CELL SURFACE SIALIC ACIDS SUCCESSFULLY MODULATE BIOLOGICAL PROCESSES}

The biosynthetic incorporation of unnatural metabolic precursors into cell surface sialic acids alters a number of cellular processes. In one study, incubation of human B-lymphoma cells or green monkey kidney epithelium cells with ManProp, ManBut, and ManPent (Figure 2) resulted in the replacement of up to $50 \%$ of their natural cell surface sialic acids with the corresponding unnatural sialic acids SiaProp, SiaBut, or SiaPent, respectively. The cells displaying the engineered sialic acid demonstrated altered susceptibility to infection by polyoma virus, a pathogen that uses sialic acid as a ligand for binding before infection $(18,21)$. This finding raises the intriguing possibility that ManNAc analogs could find a role as antiviral agents (Figure 2A). In another study, incubation of human diploid fibroblast cells with the same ManNAc analogs caused the cells to lose their sensitivity to contact inhibition of growth (45). It is interesting to note that even ManProp, the analog with the subtlest structural change, is capable of eliciting profound biological effects when incorporated into its sialic acid counterpart, SiaProp. Specifically, it is capable of selectively stimulating the proliferation of particu- lar types of cells found in the rat nervous system (38), possibly by perturbing intracellular levels of calcium (37). Furthermore, when presented in context of the tumor-associated antigen, polysialic acid (the $\alpha 2,8$-linked homopolymer of sialic acid), SiaProp is capable of stimulating the host immune system, thereby decreasing the metastatic potential of the tumor (Figure 2B) (27).

The ability to raise antibodies against unnatural sialic acid epitopes raises the intriguing possibility that they could be used as sensitive probes for the cellular presentation of these markers (Figure 2C) (24). By conjugating peptides or small molecules to an unnatural sialic acid-specific antibody, the cell surface could be endowed with novel properties that extend beyond the scope of sialic acid-mediated processes. Another practical application of antibody conjugates is their potential for the attachment of compounds to the cell surface membrane as the first step toward internalization into intracellular com

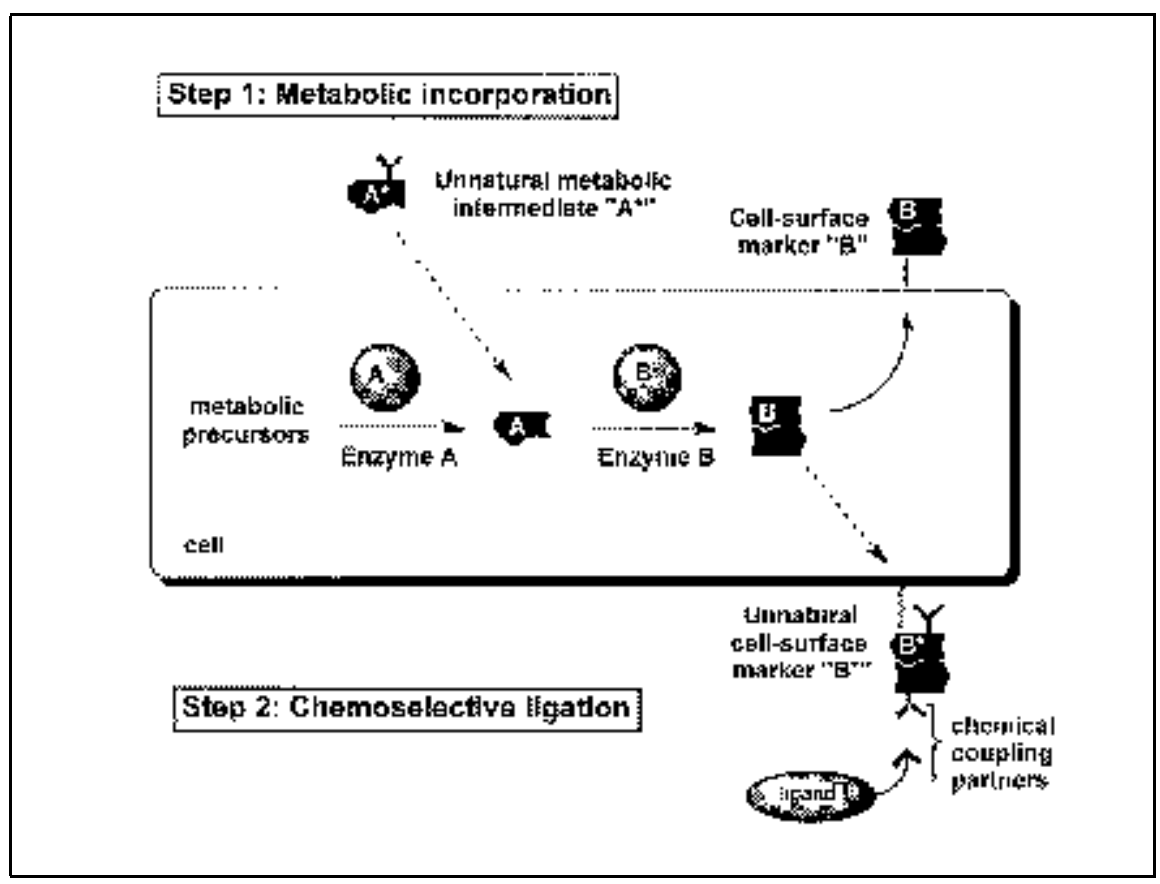

Figure 1. A substrate-based approach to cellular engineering. Substrate-based cellular engineering does not depend on the introduction of recombinant DNA or modified enzymes into a cell; instead, the naturally occurring enzymes of the cell are exploited to biosynthetically incorporate unnatural metabolic intermediates into a cellular component. In the example shown, the pathway consisting of Enzymes A and B normally converts metabolic precursors into the cell surface marker "B" (top). The first step in substrate-based engineering is intercepting this pathway with the unnatural intermediate $A^{*}$. $A *$ com petes with the endogenous substrate A for conversion to ultimately result in the presentation of the unnatural cell surface marker B* (bottom). The second step of substrate-based engineering is the chemoselective ligation of an externally delivered reagent to the molecular handle (represented by the Y-shaped moiety of $\mathrm{A}^{*}$ and $\mathrm{B}^{*}$ ) incorporated into the design of the unnatural substrate. 
partments $(32,46)$. Although their exquisite binding specificities make antibodies an attractive tool, practical limitations such as large size and cum bersome preparation urge that complementary approaches be developed. Ac- cordingly, efforts are underway to replace antibodies with small-molecule, chemical probes that will react specifically via a chemoselective ligation reaction with the metabolically incorporated, unnatural cellular component.

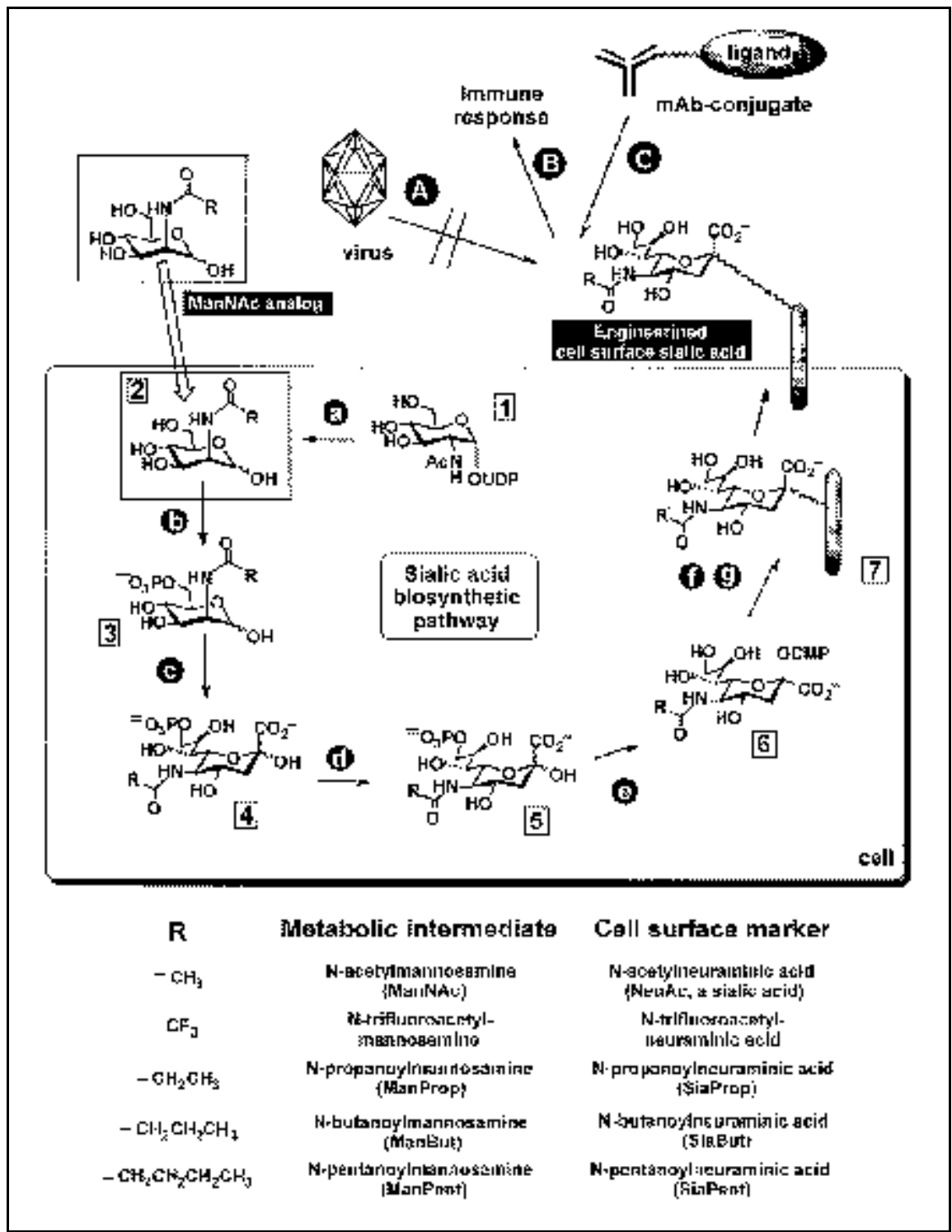

Figure 2. The sialic acid biosynthetic pathway: a model system for substrate-based cellular engineering. Exogenously supplied ManNAc analogs can intercept the sialic acid pathway and be biosynthetically processed in place of endogenously produced ManNAc. Various of these unnatural metabolic intermediates, modified at the $N$-acyl position (R, shaded, as discussed in the text), can be converted to their corresponding sialic acid counterpart and displayed on the cell surface as a terminal sugar residue of a glycoprotein or glycolipid. Applications of cell surface engineering of sialic acid residues include modulation of viral binding (A), stimulation of the immune system (B), and delivery of ligands to the cell surface by monoclonal antibody conjugates $(\mathrm{C})$. The enzymes that sequentially convert ManNAc to a sialicacid glycoconjugate are (a and b) UDP-GlcNAc 2-epimerase/ManNAc 6-kinase, (c) NeuAc synthase, (d) NeuAc 9-phosphatase, (e) CMP-NeuAc synthetase, (f) Golgi CMP-NeuAc transporter, and (g) sialyltransferase (many variants). The natural metabolic intermediates along this pathway are (no. 1) uridine diphosphate- $\mathrm{N}$-acetylglucosamine (UDP-GlcNAc); (no. 2) $\mathrm{N}$-acetylmannosamine (ManNAc); (no. 3) $\mathrm{N}$ acetylmannosamine 6-phosphate (ManNAc 6-P); (no. 4) $\mathrm{N}$-acetylneuraminic acid 9-phosphate (NeuAc 9-P); (no. 5) $\mathrm{N}$-acetylneuraminic acid (NeuAc); (no. 6) cytosine monophosphate- $N$-acetylneuraminic acid (CMP-NeuAc); and (no. 7) glycoconjugate-bound NeuAc. 


\section{REQUIREMENTS FOR CHEMOSELECTIVE LIGATION REACTIONS IN A CELLULAR ENVIRONMENT}

The concept of a "chemoselective ligation reaction" has emerged recently from the growing field of chemical biology $(23,35)$. A chemoselective ligation reaction requires two participating functional groups that have finely tuned reactivity, thereby avoiding interference with the unwanted side reactions that have the potential to occur with any other functionality that is present, even in an environment as chemically complex as the inside of a cell. The goal in developing chemoselective transformations is to equal the tremendous selectivity of non-covalent recognition events, such as antibody-antigen binding, while overcoming the limitations that are inherent in using these macromolecules as experimental tools. In the context of substrate-based cellular engineering, the goal is to incorporate one chemical functionality into a cellular component by designing it into the structure of an unnatural metabolic intermediate. A ManNAc analog is an attractive delivery vehicle for a chemical functionality because its downstream metabolic products, sialic acids, are terminal residues on cell surface glycoconjugates. They are poised on the outer periphery of the cell situated in ideal proximity for further elaboration by the attachment of an externally delivered reagent.

The development of chemoselective ligation partners for use in a physiological environment requires the addressing of several specific considerations. Ideally, the reactive partners would be abiotic, form stable adducts under physiological conditions, and recognize each other while ignoring their cellular surroundings. In addition, one of the partners should be sterically unde- manding, allowing incorporation by the cellular biosynthetic machinery. Exam ination of the unnatural mannosamine derivatives developed by the Reutter laboratory $(18,21)$ (Figure 2) suggests a simple modification to convert the inert $\mathrm{N}$-alkyl substituents into potential chemoselective coupling partners (28). Specifically, addition of a carbonyl group to the penultimate carbon of the $\mathrm{N}$-alkyl chain of ManPent, while a conservative alteration from a steric view point, provides the substrate with a new chemical functionality, the ketone:
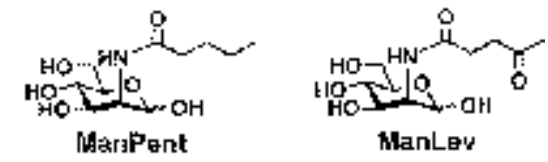

While not abiotic, ketones are relatively inert toward other compounds found within a cell and are generally absent from the cell surface. Importantly, they are electrophilic, thereby pre- 
senting a new chemical reactivity on the largely nucleophilic cell surface. Therefore, successful biosynthetic conversion of ManLev (available from Molecular Probes, Eugene, OR, USA) to its cell surface sialic acid counterpart, SiaLev, presents a potential chemoselective coupling partner, the ketone, on the cell surface $(28,48)$.

Once presented on the cell surface, keto-sialic acid residues can participate in chemoselective ligation reactions with complementary aminooxy or hydrazide functional groups (Figure 3A). Both of these nucleophiles are abiotic and unreactive towards the natural constituents of the cell surface, allowing selective ligation to metabolically incorporated ketone groups $(16,48)$. The nucleophilic coupling partner is not sterically constrained by the require- ment of enzymatic processing and can be, in theory, any peptide, carbohydrate, or small-molecule ligand com patible with physiological conditions. A particularly useful coupling partner is biotin hydrazide, which, upon further reaction with fluorescently labeled avidin (e.g., FITC-avidin), allows kinetic and quantitative evaluation of ketone presentation on the cell surface by flow cytometry (48). Human cells incubated with ManLev present SiaLev on the cell surface in a highly reproducible, dose-dependent manner with upwards of $10^{7}$ ketones accessible for reaction with the exogenous chemical probe. Maximal levels of ketones are achieved after approximately five days of incubation with ManLev and can be maintained indefinitely without adverse affects on the cell. Ketone expression is fully reversible, returning to undetectable levels three to four days after removal of ManLev from the culture medium (48).

The quantitative and kinetic analysis of SiaLev expression on the cell surface (as mentioned above and described in detail in Reference 48) provides a sensitive tool for the study of membrane recycling and glycoconjugate turnover. Additional applications of ketonebased cell surface engineering include chemical construction of new glycosylation patterns on cells (48). In theory, this technique allows any complex carbohydrate that can be constructed by chemical synthetic methods to be appended to the cell surface, thereby potentially changing the molecular identity and biological activity of the cell. Furthermore, a ManLev-based ap-

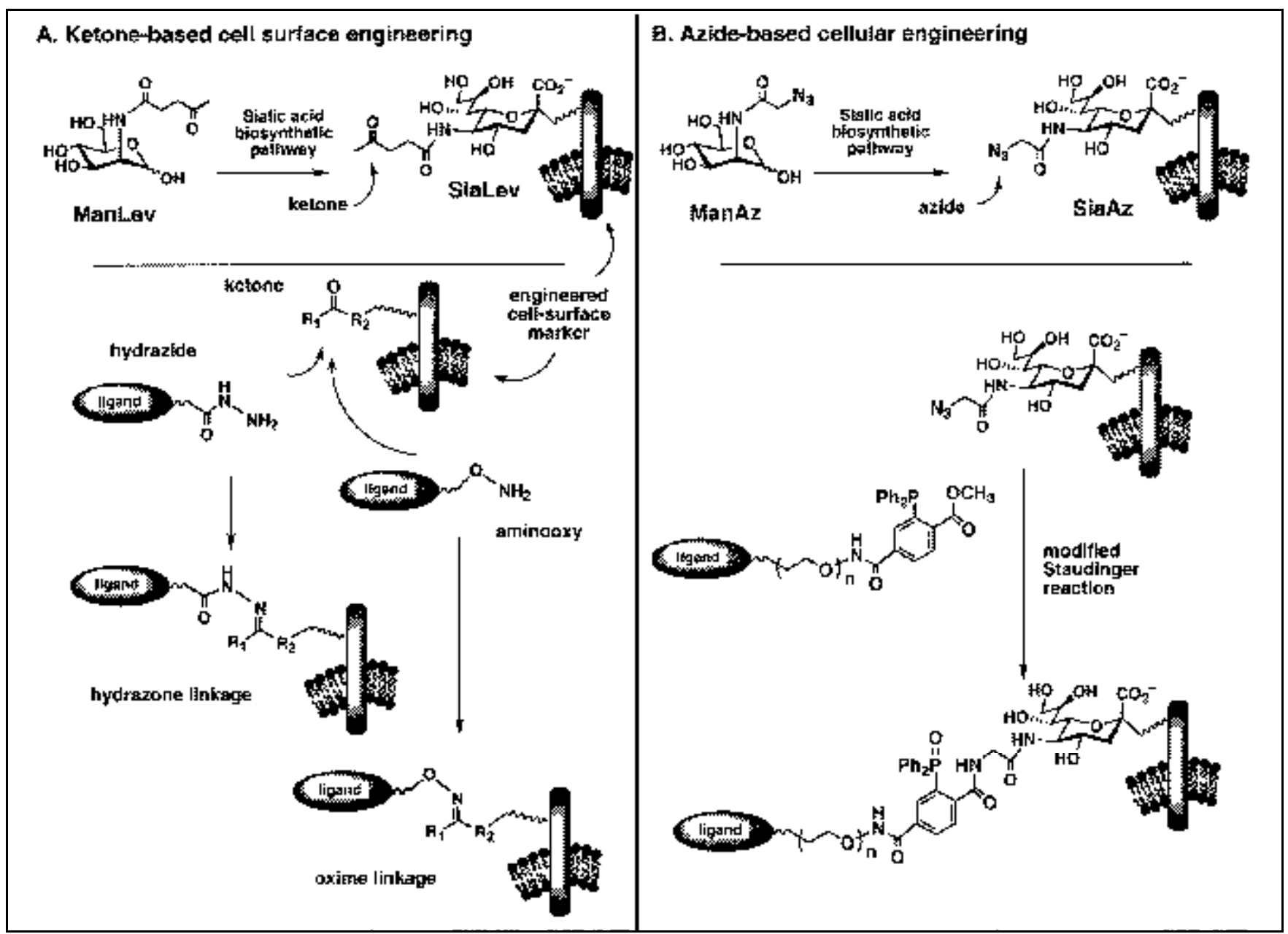

Figure 3. Chemoselective ligation reactions performed on the metabolically engineered cellular components. Chemical reactions that are compatible with physiological conditions have been developed for the coupling of ligands to either cell surface-displayed ketones (A) or azides (B). In both cases, the sialic acid pathway of a cell (see Figure 2) is exploited to convert an unnatural ManNAc analog, either ManLev or ManAz, into the corresponding cell surface displayed ketone- or azidecontaining sialic acid, respectively. Detailed explanation of these reactions is published elsewhere for both the ketone-based $(34,48)$ and azide-based $(35)$ systems. 
proach to specifically target either diagnostic (25) or therapeutic $(28,29)$ agents to tumor cells based on the aberrant sialic acid metabolism of these cells is now underway. Finally, the installation of novel receptors on cells has been demonstrated to facilitate viral-mediated gene (22).

\section{DEVELOPMENT OF ADDITION AL CHEMOSELECTIVE COUPLING PARTNERS}

Ketone ligation reactions are useful for cell surface engineering applications but have limited intracellular use because of competition with endogenous ketone-containing metabolites. Development of new cell-compatible chemoselective ligation reactions in which both coupling partners are abiotic and chemically orthogonal toward all biomolecules found within a cell and on the cell surface is desirable to fully exploit the potential of substrate-based approaches. Recently, a second chemoselective ligation reaction based on the Staudinger reaction has been reported (35). The classical Staudinger reaction occurs between a phosphine and an azide to produce an aza-ylide. These coupling partners are both completely abiotic and meet several of the criteria required for a cell-based chemoselective ligation reaction. The azide, like the ketone, is a sterically constrained functional group and would be expected to be well tolerated by the sialic acid biosynthetic machinery. The azide can be appended to the $\mathrm{N}$-acyl position of ManNAc to provide a vehicle for delivery into cellular glycoconjugates in the form of the corresponding azido-sialic acid (Figure 3B). Once incorporated into a cellular component, the azide is capable of facile chemical reaction with a phosphine to form an aza-ylide. Unfortunately, in the classical Staudinger reaction, this intermediate hydrolyzes spontaneously in an aqueous environment to yield a primary amine and phosphine oxide. This drawback necessitated the design of a phosphine coupling partner containing an appropriately situated electrophilic trap that enables rearrangement of the unstable aza-ylide to a stable amide bond (Figure 3B). This modified Staudinger re- action extends substrate-based approaches from the cell surface to the intracellular environment (35).

\section{FUTURE DIRECTIONS FOR SUB- STRATE-BASED METABOLIC AND CELLULAR ENGINEERING}

Advances in protein design and engineering, genomics, and proteomicsall combined with sophisticated mathematical algorithms and computer modeling $(9,13,17,36,40)$ - promise to provide the cellular engineer of the future with exquisite recombinant DNA-based tools for the manipulation of metabolic machinery and architecture of a cell. However, the sheer complexity of these endeavors has encouraged the exploration of alternatives to a recombinant DNA-based methodology. This report describes one such alternative, an emerging substrate-based approach in which the manipulation of small-molecule metabolites, instead of the enzymes that process these compounds, is used to achieve the goals of the metabolic or cellular engineer. The sialic acid pathway has served as a model system to illustrate the substrate-based approach. The potential biological impact of this method was demonstrated when the engineering of even very subtle structural changes into cell surface sialic acids elicited significant effects on cellular behavior. Subsequently, the development of physiologically com patible chemoselective ligation reactions now allows further chemistry to be performed on the engineered cellular component by the external delivery of a complementary reaction partner. This second step in the substrate-based metabolic engineering process has greatly increased the versatility of this emerging technology, allowing modulation of processes well beyond those strictly mediated by sialic acids.

Future expansion of substrate-based approaches in multiple directions is anticipated. First, similar exploitation of additional metabolic pathways will provide new avenues for manipulating the complex carbohydrates of the cell. Recently, cell surface expression of "ketoGal", a ketone-containing analog of $\mathrm{N}$-acetylgalactosamine (GalNAc) has been reported: 


\section{Review}
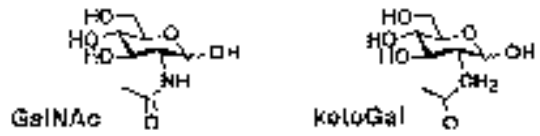

Once incorporated into a cellular glycoconjugate, the ketone moiety of ketoGal can be used as a coupling partner in chemoselective ligation reactions analogous to those described for ketone-containing sialic acids. Unlike sialic acids, residues that occur at the outer termini of complex carbohydrates, GalNAc is situated at an internal position, proximal to the peptide chain in $O$-linked glycoconjugates, as well as in the repeating units of the polymeric oligosaccharide chondroitan sulfate (12). Clearly, the ongoing development of metabolic analogs for presentation in a variety of structural contexts that are beyond the access of the sialic acid pathway significantly broadens the scope of substratebased engineering approaches.
Finally, this review is focused on engineering unnatural substituents into the carbohydrate component of cells by exploiting the substrate permissivity of the naturally occurring enzymes of the cell. Future efforts will be complemented by directed evolution methods that allow enzymes to be custom designed to process unnatural sugars that are currently unpalatable for the native enzymes $(8,49)$. A challenge will be to integrate these designer enzymes into the metabolic machinery of a cell, thereby creating entire pathways with novel substrate specificity. The synthesis of chemical and genetic approaches will provide a powerful tool to direct the construction of cellular components with well-defined oligosaccharide landscapes of novel composition (47). It is worth noting that efforts to com bine chemistry and genetics are not exclusive to the field of glycobiology. In particular, protein engineers are cur- rently exploring the use of unnatural substrates in organisms harboring expanded genetic repertoires in their quest to incorporate amino acid analogs into the macromolecular architecture of a cell $(26,42)$. In conclusion, substratebased approaches that allow metabolic incorporation of unnatural components into cellular components are a welcome new tool for the cellular engineer.

\section{REFERENCES}

1.Atkinson, P. H. and J. Hakimi. 1980. Alterations in glycoproteins of the cell surface, $p$. 191-239. In W.J. Lennarz (Ed.), The Biochem istry of Glycoproteins and Proteoglycans. Plenum Press, New York.

2.Bailey, J.E. 1991. Toward a science of metabolic engineering. Science 252:1668-1675.

3.Bernacki, R.J. and W. Korytnyk. 1982. Development of membrane sugar and nucleotide sugar analogs as potential inhibitors or modifiers of cellular glycoconjugates, p. 245-263. In M.I. Horowitz (Ed.), The Glycoconjugates 
(IV). Academic Press, New York.

4.Borst, P. and A.H. Schinkel. 1997. Genetic dissection of the function of mammalian P-glycoproteins. Trends Genet. 13:217-222.

5.Cameron, D.C. and I.T. Tong. 1993. Cellular and metabolic engineering. An overview. Appl. Biochem. Biotechnol. 38:105-140.

6.Cashman, J.R., B.Y. Perotti, C.E. Berkman, and J. Lin. 1996. Pharmacokinetics and molecular detoxication. Environ. Health Perspect. 104:23-40.

7.Dhakshinamoorthy, S., D.J. Long, 2nd, and A.K. Jaiswal. 2000. Antioxidant regulation of genes encoding enzymes that detoxify xenobiotics and carcinogens. Curr. Top. Cell. Regul. 36:210-216.

8.Fong, S., T.D. Machajewski, C.C. Mak, and C.-H. Wong. 2000. Directed evolution of D-2keto-3-deoxy-6-phosphogluconate aldolase to new variants for the efficient synthesis of Dand L-sugars. Chem. Biol. 7:873-883.

9.Gombert, A.K. and J. Nielsen. 2000. Mathematical modelling of metabolism. Curr. Opin. Biotechnol. 11:180-186.

10.Goon, S. and C.R. Bertozzi. 2001. Metabolic substrate engineering as a tool for glycobiology, p. 641-674. In P.G. Wang and C.R. Bertozzi (Eds.), Glycobiology: Principles, Synthesis, and Applications. Marcel Dekker, New York.

11.Grunholz, H.-J., E. Harms, M. Opetz, W. Reutter, and M. Cerny. 1981. Inhibition of in vitro biosynthesis of $\mathrm{N}$-acetylneuraminic acid by $N$-acyl- and $N$-alkyl-2-amino-2-deoxyhexoses. Carbohydr. Res. 96:259-270.

12.Hang, H.C. and C.R. Bertozzi. 2001. Ketone isosteres of 2- $N$-acetamindosugars as substrates for metabolic cell surface engineering. J. Am. Chem. Soc. 123:1242-1243.

13.Hatzimanikatis, V., L.H. Choe, and K.H. Lee. 1999. Proteomics: theoretical and experimental considerations. Biotechnol. Prog. 15:312-318.

14.Hipfner, D.R., R.G. Deeley, and S.P. Cole. 1999. Structural, mechanistic, and clinical aspects of MRP1. Biochim. Biophys. Acta 1461:359-376

15.Hynes, R.O. 1974. Role of surface alterations in cell transformation: the importance of proteases and surface proteins. Cell 1:147-156.

16.Jacobs, C.L., K.J. Yarema, L.K. Mahal, D.A. Nauman, N.W. Charters, and C.R. Bertozzi. 2000. Metabolic labeling of glycoproteins with chemical tags through unnatural sialic acid biosynthesis. Methods Enzymol. 327:260-275.

17.Kao, C.M. 1999. Functional genomic technologies: creating new paradigms for fundamental and applied biology. Biotechnol. Prog. 15:304-311.

18.Kayser, H., R. Zeitler, C. Kannicht, D. Grunow, R. Nuck, and W. Reutter. 1992 Biosynthesis of a nonphysiological sialic acid in different rat organs using $N$-propanoyl-D hexosamines as precursors. J. Biol. Chem. 267:16934-16938

19. Kelm, S. and R. Schauer. 1997. Sialic acids in molecular and cellular interactions. Int. Rev. Cytol. 175:137-240.

20.Keppler, D. 1999. Export pumps for glutathione S-conjugates. Free Radic. Biol. Med. 27:985-991.

21.Keppler, O.T., P. Stehling, M. Herrmann, H. Kayser, D. Grunow, W. Reutter, and $M$.
Pawlita. 1995. Biosynthetic modulation of sialic acid-dependent virus-receptor interactions of two primate polyoma viruses. J. Biol. Chem. 270:1308-1314.

22.Lee, J.H., T.J. Baker, L.K. Mahal, J. Zabner, C.R. Bertozzi, D.F. Wiemer, and M.J. Welsh. 1999. Engineering novel cell surface receptors for virus-mediated gene transfer. J. Biol. Chem. 31:21878-21884.

23.Lemieux, G.A. and C.R. Bertozzi. 1998 Chemoselective ligation reactions with proteins, oligosaccharides and cells. Trends Biotechnol. 12:506-513.

24.Lemieux, G.A. and C.R. Bertozzi. 2001 Modulating cell surface immunoreactivity by metabolic induction of unnatural carbohydrate antigens. Chem. Biol. 8:265-275.

25.Lemieux, G.A., K.J. Yarema, C.L. Jacobs, and C.R. Bertozzi. 1999. Exploiting differences in sialoside expression for selective targeting of MRI contrast reagents. J. Am. Chem. Soc. 121:4278-4280.

26.Liu, D.R. and P.G. Schultz. 1999. Progress toward the evolution of an organism with an expanded genetic code. Proc. Natl. Acad. Sci. USA 96:4780-4785.

27.Liu, T., Z. Guo, Q. Yang, S. Sad, and H.J. Jennings. 2000. Biochemical engineering of surface $\alpha$ 2-8 polysialic acid for immunotargeting tumor cells. J. Biol. Chem. 275:32832 32836 .

28.Mahal, L.K., K.J. Yarema, and C.R. Bertozzi. 1997. Engineering chemical reactivity on cell surfaces through oligosaccharide biosynthesis. Science 276:1125-1128.

29.Mahal, L.K., K.J. Yarema, G.A. Lemieux, and C.R. Bertozzi. 1999. Chemical approaches to glycobiology: engineering cell surface sialic acids for tumor targeting, p. 267-280. In Y. Inoue, Y.C. Lee, and F.A. Troy II, (Eds.), Sialobiology and Other Novel Forms of Glycosylation. Gakushin Publishing, Osaka, Japan.

30.Martin, A., C. Rambal, V. Berger, S. Perier, and P. Louisot. 1998. Availability of specific sugars for glycoconjugate biosynthesis: a need for further investigations in man. Biochimie 80:75-86.

31.Meyer, U.A. 1996. Overview of enzymes of drug metabolism. J. Pharmacokinet. Biopharm. 24:449-459.

32.Mukherjee, S., R.N. Ghosh, F.R. Maxfield. 1997. Endocytosis. Physiol. Rev. 77:759-803.

33.Paul, B., R.J. Bernacki, and W. Korytnyk. 1980. Synthesis and biological activity of some 1 - $N$-substituted 2-acetamido-2-deoxy- $\beta$-D-glycopyranosylamine derivatives and related analogs. Carbohydr. Res. 80:99-115.

34. Rideout, D., T. Calogeropoulou, J. Jaworski, and M. McCarthy. 1990. Synergism through direct covalent bonding between agents: a strategy for rational design of chemotherapeutic combinations. Biopolymers 29:247-262.

35.Saxon, E. and C.R. Bertozzi. 2000. Cell surface engineering by a modified Staudinger reaction. Science 287:2007-2010

36.Schilling, C.H., S. Schuster, B.O. Palsson, and R. Heinrich. 1999. Metabolic pathway analysis: basic concepts and scientific applications in the post-genomics era. Biotechnol. Prog. 15:296-303.

37.Schmidt, C., C. Ohlemeyer, H. Kettenmann, W. Reutter, and R. Horstkorte. 2000. Incor- poration of $\mathrm{N}$-propanoylneuraminic acid leads to calcium oscillations in oligodendrocytes upon the application of GABA. FEBS Lett. 478:276-280.

38.Schmidt, C., P. Stehling, J. Schnitzer, W. Reutter, and R. Horstkorte. 1998. Biochemical engineering of neural cell surfaces by the synthetic $N$-propanoyl-substituted neuraminic acid precursor. J. Biol. Chem. 273:1914619152.

39.Schultz, A.M. and P.T. Mora. 1975. Inhibition of the metabolism of amino sugars with 2-deoxy-2-(2-fluoroacetamido)- $\alpha$-D-glucopyranose. Carbohydr. Res. 40:119-127.

40.Schuster, S., D.A. Fell, and T. Dandekar. 2000. A general definition of metabolic pathways useful for systematic organization and analysis of complex metabolic networks. Nat. Biotechnol. 18:326-333.

41.Schwartz, E.L., A.F. Hadfield, A.E. Brown, and A.C. Sartorelli. 1983. Modification of sialic acid metabolism of murine erythroleukemia cells by analogs of $N$-acetylmannosamine. Biochim. Biophys. Acta 762:489497.

42.Sharma, N., R. Furter, P. Kast, and D.A. Tirrell. 2000. Efficient introduction of aryl bromide functionality into proteins in vitro. FEBS Lett. 467:37-40.

43.Sillanaukee, P., M. Ponnio, and I.P. Jaaskelainen. 1999. Occurrence of sialic acids in healthy humans and different disorders. Eur. J. Clin. Invest. 29:413-425

44.Varki, A. 1997. Sialic acids as ligands in recognition phenomena. FASEB J. 11:248-255.

45.Wieser, J.R., A. Heisner, P. Stehling, F. Oesch, and W. Reutter. 1996. In vivo modulated $\mathrm{N}$-acyl side chain of $\mathrm{N}$-acetylneuraminic acid modulates the cell contact-dependent inhibition of growth. FEBS Lett. 395:170-173.

46.Wojda, U., P. Goldsmith, and J.L. Miller. 1999. Surface membrane biotinylation efficiently mediates the endocytosis of avidin bioconjugates into nucleated cells. Bioconjug. Chem. 10:1044-1050.

47.Yarema, K.J. and C.R. Bertozzi. 1998 Chemical approaches to glycobiology and emerging carbohydrate-based therapeutic agents. Curr. Opin. Chem. Biol. 2:49-61.

48. Yarema, K.J., L.K. Mahal, R.E. Bruehl, E.C. Rodriguez, and C.R. Bertozzi. 1998. Metabolic delivery of ketone groups to sialic acid residues. Applications to cell surface glycoform engineering. J. Biol. Chem. 273:3116831179 .

49.Zhang, J.H., G. Dawes, and W.P. Stemmer. 1997. Directed evolution of a fucosidase from a galactosidase by DNA shuffling and screening. Proc. Natl. Acad. Sci. USA 94:4504-4509.

Address correspondence to:

Dr. Kevin J. Yarema

Whitaker Biomedical Engineering Institute

Whiting School of Engineering

Johns Hopkins University

Baltimore, MD 21218, USA

e-mail: kjyarema@bme.jhu.edu 